\title{
Síntomas óticos referidos en desórdenes temporomandibulares. Relación con músculos masticatorios
}

\author{
Luis Miguel Ramírez ${ }^{1}$, Luis Ernesto Ballesteros ${ }^{1}$, G ermán $^{2}$ \\ Pablo Sandoval ${ }^{2}$ \\ O tological symptoms among patients \\ with temporomandibular joint \\ disorders
}

Middle ear muscles have a common embryological and functional origin with masticatory and facial muscles. Therefore, symptoms referred to the ear may originate from the stomatognathic area. When a primary otological cause is discarded in the diagnostic work up for tinnitus, vertigo, hypoacousia, byperacousia, ear pain or sensation of occluded ear, a temporomandibular joint dysfunction may be the cause of these symptoms. Temporomandibular joint dysfunction is twice more common among women and has environmental, physiological and behavioral causes. Among patients with this dysfunction, the prevalence of ear pain, tinnitus and dizziness varies between 33 and $76 \%$ (Rev Méd Chile 2007; 135: 1582-90).

(Key words: Dizziness; Temporomandibular joint disorders; Tinnitus)

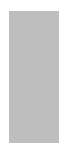

Recibido el 18 de agosto, 2006. Aceptado el 14 de diciembre, 2006.

${ }^{1}$ Departamento de Ciencias Básicas, Universidad Industrial de Santander. Bucaramanga, Colombia. ${ }^{2}$ Departamento de Cirugía, Universidad Industrial de Santander. Bucaramanga, Colombia.

$\mathrm{L}$ a mayoría de los personas con desórdenes temporomandibulares (DTM) sufren de dolor muscular crónico de tipo local, que afectan los músculos orofaciales y también pueden producir dolor y sintomatología no dolorosa de tipo referida, que puede llegar a afectar la musculatura cervical y la musculatura del oído medio, con síntomas óticos variados, que incluyen vértigo,

Correspondencia $a$ : Dr. Luis Miguel Ramirez, Fax: (097) 6479668. E mail: lmra3@yahoo.com tinitus, sensación de ó́do tapado, hiper o hipoacusia y otalgia. Múltiples posibilidades anatómicas o neurológicas que, a partir de una disfunción muscular o articular, pueden generar condiciones óticas, no parecieran corresponder con los hallazgos clínicos al momento de la valoración $^{1}$. Estas posibles vías atañen tanto el enfoque puramente descriptivo de estructuras anatómicas en común para la articulación temporomandibular (ATM) y el oído, así como también vecindades entre estructuras musculares y el oído medio $^{2}$. Esta revisión pretende desarrollar bajo un enfoque principalmente anatómico y neuroanató- 
mico las conexiones entre el oído y el sistema estomatognático. Su objetivo se centra en la comprensión de la sintomatología ótica sin origen ótico, en la racionalización del vínculo músculoesquelético, bajo una perspectiva anatómica y en la integración de disciplinas en salud que comparten síntomas comunes.

\section{REVISIÓN}

La actividad muscular normal del músculo tensor del tímpano (músculo masticatorio) y el músculo estapedial, está relacionada con un mecanismo neurológico reflejo conocido como "la vía centrífuga" en los mecanismos nerviosos de protección al trauma sonoro y la discriminación auditiva (de tonos bajos), además de una compleja co-activación durante los movimientos velo-faríngeos. La actividad anormal del músculo tensor del tímpano (aparentemente durante los DTM) está asociada a síntomas óticos expresados como sensación de oído tapado, tinitus, vértigo e hipo-hiperacusia, sin existir una enfermedad ótica que se muestre con uno a más de estos síntomas, como en el síndrome de Ménière, otitis, neurinoma del ángulo pontocerebeloso u otosclerosis, entre otros. Los cambios en la actividad muscular intratimpánica producen cambios en la impedancia aérea y líquida del oído medio e interno, respectivamente, que pueden ser medidos por impedanciometría y audiometría.

Los DTM son una sub-clasificación de los desórdenes músculo esqueléticos e incluyen una amplia serie de condiciones craneofaciales, con etiología multifactorial, tanto en adultos como en niños ${ }^{3}$. Klockhoff y col. prueban la tensión simultánea de los músculos tensor del tímpano y temporal electromiográficamente, lo que trae una relación afín entre la tensión de los músculos orofaciales, del oído y masticatorios por un mayor tono simpático durante el estrés emocional y DTM que comparten vías fisiopatológicas comunes ${ }^{4}$.

La tensión emocional incrementada genera actividad muscular masticatoria disfuncional y consecuentemente DTM. Lam y col. ${ }^{5}$ afirman que la prevalencia de otalgia y demás síntomas de causas no otológicas en pacientes con DTM varía entre $3,5 \%$ y $42 \%$ y que la prevalencia de tinitus en pacientes con DTM, parece ser mayor que la encontrada en la población general ${ }^{6}$. La frecuen- cia de tinitus en pacientes con DTM varía de 33\% a $76 \% 7^{7,8}$. La prevalencia de mareos en pacientes con DTM es del orden de $40 \%$ a $70 \%$ y el vértigo de $5 \%$ a $40 \% 9,10$. Aquí es importante señalar las limitaciones metodológicas de los estudios realizados hasta este momento e igualmente sus discrepancias, que generan sesgos por los criterios de diagnóstico y una difícil interpretación de los resultados por la ausencia de estandarización. La prevalencia de los DTM es dos a nueve veces mayor en mujeres que en hombres. Estas son patologías complejas, con consecuencias que van desde la dificultad para masticar y otras funciones orales, hasta el dolor agudo, subagudo y crónico. La etiología de los DTM se puede resumir en el macrotrauma y microtrauma (bruxismo). El bruxismo juega un rol importante en los DTM y en los síntomas orofaciales referidos, aunque muchas investigaciones consideran esta asociación inconclusa ${ }^{11}$. El nexo íntimo entre la actividad masticatoria disfuncional como el bruxismo, los DTM y el estrés emocional, abren la relación causa-efecto entre ellos. La etiología de los DTM tiene un origen multifactorial que involucra agentes ambientales, fisiológicos y de conducta.

Los DTM producen tensión y contracción de los músculos masticatorios y contracción refleja en el músculo tensor del velo palatino (peri-estafilino externo) y tensor del tímpano (tensor del martillo) por la inervación motora común de la rama mandibular del trigémino en estos músculos ${ }^{12-17}$. Schames y col. ${ }^{18}$ igualmente afirman que la disfunción del tensor del tímpano y el tensor del velo del paladar, juega un papel importante en la relación de los DTM y los síntomas óticos y los denominan músculos accesorios de la masticación por su innervación motora común del trigemino y por su funcionalidad.

Entre los síntomas óticos, la otalgia puede llegar a producirse por la tensión de la membrana timpánica en el aumento de la tonicidad del tensor del tímpano debido a su inserción en el martillo y éste, a su vez, en la membrana timpánica. La disacusia es otro síntoma que viene acompañado de sensaciones acústicas extrañas o alucinatorias como el tinitus, ya que sólo el que las sufre las oye y pueden percibirse como grillos, clicks, pops, susurros o ruidos de rozamiento. Esta tensión muscular en el oído medio también puede estar asociada a hipo o hiperacusia de origen conducti- 
vo y presentación subjetiva (aún con valores audiométricos normales). La tensión física incrementada por estrés emocional y las cefaleas acompañan concomitantemente esta sintomatología ótica.

La interacción neuromuscular compleja entre los músculos de la masticación y el oído, a través del músculo tensor del tímpano, fue observada por Klockhoff en $1961^{19}$, pero fue denominada "síndrome otognático" por Myrhaug ${ }^{13}$, hasta 1964 y posteriormente "síndrome otomandibular" por Bernstein, en $1969^{14}$ y por Arlen en $1977^{20}$. Los pacientes con síndrome otomandibular presentan uno o más síntomas óticos, sin patología localizada en oído, nariz o garganta, pero con uno o más músculos de la masticación en estado de constante espasmo. Ogutcen-Toller y Juniper ${ }^{21}$ notaron que las estructuras que conducen el sonido en el oído medio, pueden ser afectadas por la contracción refleja del tensor del tímpano por DTM. Aunque sugieren al espasmo reflejo como causa de síntomas óticos, realizaron la evaluación audiológica en pacientes con DTM y síntomas óticos sin encontrar una franca disfunción del oído medio y la trompa de Eustaquio, sugiriendo más bien una disminución auditiva de tipo subjetiva (conducti- va), modulada en la actividad disfuncional del músculo tensor del tímpano. Travell y Simons ${ }^{22}$ explican el tinitus como un fenómeno sensitivo referido o un espasmo muscular reflejo de los músculos tensores del tímpano y estapedial en el oído medio.

Myrhaug $^{13}$ afirmó que en el tinitus subjetivo hay contracción refleja del músculo tensor del tímpano y que en el tinitus objetivo (también oído por el médico), en donde hay una apertura y cierre rítmicos del área faríngea de la trompa de Eustaquio (mioclonus palatino), la contracción muscular refleja está presente en el tensor del tímpano y el tensor del velo palatino ${ }^{23-26}$. Zipfel y col. $^{27}$ amplían lo anterior, explicando que las causas mecánicas más comunes del tinitus objetivo son el mioclonus palatino y el mioclonus del oído medio, que produce movimiento rítmico de la membrana timpánica (30 veces por minuto), secundaria a la contracción repetitiva de los músculos tensor del tímpano y estapedial (Figura 1). También declaran que el tinitus objetivo (excluyendo anormalidades vasculares) es mucho menos común que el tinitus subjetivo ${ }^{28-30}$. Este comportamiento muscular puede corresponder a un patrón reflejo por fatiga, originada en el funciona-

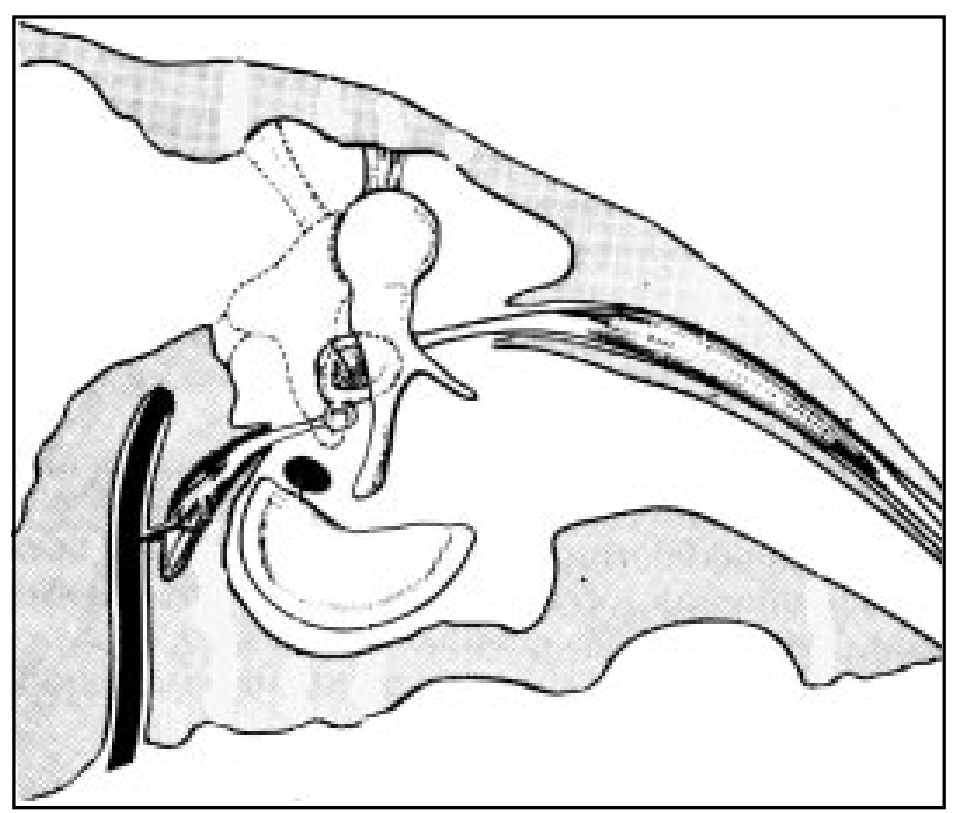

Figura 1. Músculo tensor del tímpano y músculo estapedial en la cavidad timpánica. Modificado de Ref. 12. 
Tabla I. M ovimientos en común de músculos intra y extratimpánicos

\begin{tabular}{|lccc|}
\hline Músculos & Tensor del tímpano & Tensor del Velo Palatino & Estapedial \\
\hline Movimientos & Inervación: Trigémino & Inervación: Trigémino & Inervación: Facial \\
\hline Hablar & $\mathrm{x}$ & $\mathrm{x}$ & \\
Masticar & $\mathrm{x}$ & $\mathrm{x}$ & \\
Tragar & $\mathrm{x}$ & $\mathrm{x}$ & \\
Bostezar & $\mathrm{x}$ & $\mathrm{x}$ & \\
Reír & $\mathrm{x}$ & $\mathrm{x}$ & \\
Estornudar & $\mathrm{x}$ & $\mathrm{x}$ & \\
Inspiración & $\mathrm{x}$ & $\mathrm{x}$ & $\mathrm{x}$ \\
Trauma sonoro & $\mathrm{x}$ & & $\mathrm{x}$ \\
Antes de hablar & $\mathrm{x}$ & $\mathrm{x}$ & $\mathrm{x}$ \\
Reflejo palpebral & $\mathrm{x}$ & & \\
\hline
\end{tabular}

miento somatomotor anormal de estos músculos durante la tensión emocional aumentada y fases de bruxismo ${ }^{32}$. Klockhoff y col. ${ }^{4}$ consideraron como signo patognomónico, de la actividad muscular disfuncional intratimpánica (por DTM) la "labilidad impedanciométrica anormal" que puede presentarse con "ataques" de síntomas óticos repentinos y fluctuantes que incluyen tinitus, sensación de oído tapado e hipo o hiperacusia subjetiva súbita y paroxística. Pau y col. ${ }^{37}$ muestran lo anterior a través de audiogramas y denominan esto como un fenómeno inducido por el tensor del tímpano, que puede llegar a emular perdidas sensorineuronales (conducción ósea) por la rigidez de la cadena oscicular que produce esta actividad muscular anormal.

El músculo tensor del tímpano presenta un umbral alto (100 dB), una latencia de $12 \mathrm{~ms}$ y una amplitud grande y variable, que puede ser enmascarada por el músculo estapedial en pruebas de impedancia, debido a su menor umbral $(70 \mathrm{~dB})$ y latencia $(7 \mathrm{~ms})$. A través de electromiografía y pruebas genéticas, en otras especies se ha observado, igual que el músculo estapedial, el músculo tensor del tímpano ha mostrado ser un músculo con propiedades contráctiles especiales y resistente a la fatiga debido a su composición de fibras glicoliticas oxidativas rápidas, lo que se ajusta a la demanda mecánica del estimulo acústico, respondiendo de manera constante e inmediata al estimulo acústico de intensidad entre 70-100 dB y frecuencia de $2 \mathrm{KHz}^{31,32}$. Según Ochi y $\mathrm{col}^{33}$, la actividad del músculo tensor del tímpano, además del reflejo centrífugo originado en la coclea, puede ser evocada igualmente desde orígenes somato-sensoriales o sensori-vestibulares. Las aferencias sensoriales de sitios periféricos (inervados por el trigémino) como la membrana timpánica (por la estimulación de la energía acústica) o la corneal (reflejo corneal), evocan la actividad del músculo tensor del tímpano y, a su vez, una deflexión invertida ipsilateral de la membrana timpánica (reflejo acústico ipsilateral invertido). La estimulación vestibular por la contracción del tensor del tímpano, genera del mismo modo potenciales miogénicos a partir de receptores saculares estimulados por la sostenida presión perilinfática del estribo ${ }^{34-35}$.

Shapiro y Truex ${ }^{36}$ sugirieron un posible espasmo tónico por contracción refleja del tensor del tímpano y del músculo estapedial al ser irritados periféricamente el V y VII par, respectivamente, que puede originar una pérdida o una disminución de la habilidad auditiva en los tonos bajos y que, a diferencia del mioclonus intratimpánico, produce un estado de contracción tónica y sostenida que puede fluctuar con efectos óticos episódicos. Esta irritación puede originarse en los desórdenes crónicos músculo-esqueléticos como los DTM. En normalidad, el equilibrio de la cadena oscicular y el normal funcionamiento de las estructuras que conducen el sonido en el oído medio dependen del músculo tensor del tímpano y estapedial (músculos antagónicos) ${ }^{37}$. En DTM, la 
contracción sostenida y refleja de estos músculos puede alterar la presión peri y endolinfática a través de los cambios transmitidos desde la ventana oval hacia las paredes del laberinto y canales semicirculares, que pueden desencadenar un desbalance de los impulsos vestibulares, de la recepción sensorial acústica y una reacción sintomática similar al síndrome de Menière ${ }^{38}$.

Aquí es imperativo reconocer a la cadena oscicular (martillo, yunque y estribo) bajo una biomecánica eficiente pero frágil dentro del oído medio, ya que están prácticamente sostenidos de una manera débil por estructuras como la membrana timpánica, algunos ligamentos y los tendones de los músculos tensor del tímpano y estapedial que sostienen estos huesos en una eficaz y adaptada biomecánica al estímulo de la energía sonora. La función de los músculos auditivos (tensor del tímpano, tensor del velo palatino y estapedial) se relacionan en el Tabla I. La contracción del músculo estapedial se produce con sonidos fuertes e inmediatamente antes de hablar, mejorando la discriminación auditiva, vocalización externa y reduciendo el efecto enmascarador del sonido autógeno ${ }^{39-40}$. El músculo tensor del tímpano, a pesar de lo que comúnmente se cree, no es un músculo inoperante, ya que responde electromiográficamente a sonidos fuertes y estí- mulos externos activados por la vocalización, la masticación, la deglución y el movimiento de los músculos faciales ${ }^{41}$. Si los músculos tensor del velo palatino y tensor del tímpano comparten movimientos en común (velo-faríngeos y faciales), y el tensor del tímpano junto con el músculo estapedial son activados neurologicamente en reflejos de protección auditiva al trauma acústico y discriminación auditiva (vía centrifuga muscular), no es aventurado afirmar que los movimientos faciales y velo-faríngeos compartidos por los dos tensores, tengan una ingerencia importante en los síntomas óticos episódicos debido a su inserción en el maléolo de la cadena oscicular.

Rood y Doyle ${ }^{42,43}$, como resultado de disecciones cadavéricas en adultos y fetos, encuentran la anatomía del músculo tensor del velo palatino compuesta por una porción medial o "dilatador de la trompa" -descrita por Gray en 1918-, una porción externa compuesta por el músculo tensor del velo palatino y una intratimpánica compuesta por el músculo tensor del tímpano que entremezcla sus fibras con la zona externa del músculo tensor del velo palatino. La zona lateral del músculo tensor del velo palatino se origina en la espina del esfenoides, fosa escafoidea, músculo tensor del tímpano y reborde óseo lateral del surco esfenoideo. La zona medial del tensor del

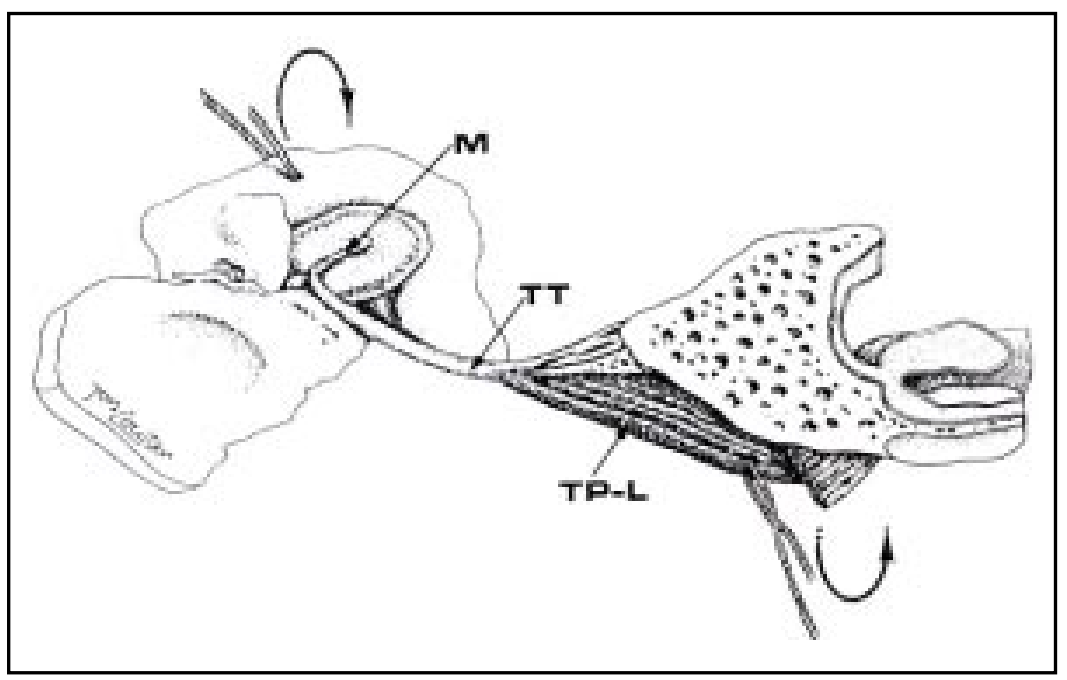

Figura 2. Conexión de las fibras del músculo tensor del tímpano y tensor del velo palatino. $M$. Manubrio del martillo, TT. Músculo Tensor del Tímpano, TP-L. Músculo Tensor del Velo Palatino. Tomado con permiso de Ref. 28. 
velo palatino se origina en el tercio posterior y medio de la trompa de Eustaquio, en su pared membranosa lateral. Estos músculos descienden y convergen al hamulus pterigoideo en un fuerte tendón, que se curva alrededor de este proceso para insertarse de manera horizontal en el paladar blando como aponeurosis palatina. Estos autores afirman que los patrones de movimiento como el bostezo, reír, tragar y toser involucran músculos faríngeos y laríngeos que activan el músculo tensor del tímpano.

Kamerer ${ }^{40}$, en 1978, expresó su extrañeza ante la no creación de una teoría unificada para estos músculos que comparten una relación anatómica estrecha y que probó electromiográficamente cuando el tensor del tímpano y el tensor del velo palatino funcionaron simultáneamente durante la deglución, generando la bomba de aire en el oído medio sugerida por Djupesland que asiste a la ventilación tubárica ${ }^{44}$. Explicó cómo el tensor del tímpano, durante su contracción recíproca con el tensor del velo palatino, genera una deflexión interna de la membrana timpánica que rompe el sello entre la membranas mucosas del istmo de la trompa de Eustaquio por el aire expulsado, contribuyendo a la ventilación del oído medio ${ }^{45}$. Barsoumian y col. ${ }^{46}$ corroboraron los hallazgos de Lupin en 1969 y posteriormente los de Rood y Doyle, encontrando en cadáveres adultos que las fibras de la zona más externa del músculo tensor del velo palatino y las fibras del músculo tensor del tímpano, se unen en el oído medio en una pequeña porción tendinosa, mostrando una conexión anatómica en el funcionamiento de estos músculos (Figura 2). Los músculos tensor del tímpano y tensor del velo palatino actúan simultáneamente de manera sinérgica y pueden aumentar de manera temporal la presión intratimpánica.

No es aventurado afirmar, entonces, que el tensor del velo palatino tiene un origen óseo adicional en el manubrio del martillo. La disfunción del músculo tensor del velo palatino en DTM o durante fases de bruxismo puede modificar la posición medial del martillo y la membrana timpánica de manera individual o combinada por el anclaje del músculo tensor del tímpano. Igualmente, la disposición espacial de la cadena oscicular puede ser modificada por la tensión del músculo tensor del tímpano, por su continuidad con el músculo tensor del velo palatino. Kierner y col. ${ }^{47}$, en 2002, encuentran la conexión funcional entre el tensor del velo palatino y el músculo tensor del tímpano a través de cortes histológicos en cinco cadáveres humanos. Afirman que la reconfirmación de este hallazgo representa un paso importante en el entendimiento de la unidad funcional entre estos dos músculos en el hombre. Sumado a lo anterior, Schames y Col. ${ }^{18}$ sugieren la contractura miofibrótica secundaria a adhesiones y puntos gatillo de los músculos tensor del velo palatino y tensor del tímpano por disfunción generada en DTM que complica la relación anatómica de éstos y la sintomatología ótica por reducción en la longitud de sus fibras musculares.

Para finalizar, es importante comprender que la fisiología de la aireación del oído medio a través de la trompa de Eustaquio, involucra territorios neurológicos reflejos del sistema masticatorio. Malkin ${ }^{48}$, en 1987, expuso que el músculo tensor del tímpano sirve como gatillo barorreceptor al cumplir una función propioceptora a partir de su longitud muscular, que puede modificarse por hipotonía durante las presiones bajas de la cavidad timpánica (intercambio gaseoso) que retraen medialmente la membrana timpánica y, a su vez, el martillo por la mayor presión ambiental externa. Este movimiento maleolar hace hipotónico este músculo y a partir de sus usos musculares dispara un mecanismo reflejo al núcleo motor del trigémino, iniciando la contracción de este músculo y del tensor del velo palatino, lo que redunda en la apertura de la trompa de Eustaquio y la aireación de la cavidad timpánica. Estos mecanismos fisiológicos normales pueden verse obstaculizados por la hipertonía del músculo tensor del tímpano durante un DTM, lo que se expresaría en una disfunción tubárica y la sintomatología acompañante de ésta: hipo e hiperacusia subjetiva, tinitus, vértigo, otalgia, sensación de oído tapado e inclusive otitis medias.

Durante casi un siglo, diferentes investigadores han intentado darle forma y una relación de causa-efecto a los síntomas óticos a partir de los DTM bajo una perspectiva mecánica, vascular, neurológica, muscular y embriológica evidente. El oído y el sistema masticatorio fueron separados por la medicina y la odontología durante su conformación, situación que ha sido discretamen- 
te subsanada con especialidades como la otorrinolaringología y el dolor craneofacial, que cada vez cierran más la brecha de esta temática que ya iba adquiriendo la aridez de un "terreno de nadie".

\section{ConClusión}

Este artículo fue desarrollado por la necesidad de comprender la presencia de músculos masticatorios en el oído medio que puede complicar la sintomatología ótica en presencia de un DTM, entre otras causas estomatognáticas de tipo neurológico y mecánico. La anatomía, neuroanatomía y fisiología de estos músculos, además de interesante por sí misma, se relaciona con una actividad intratimpánica de modulación masticatoria externa que se vera implicada en presencia de desórdenes músculo esqueléticos del sistema estomatognático.

\section{REFERENCIAS}

1. Ramirez LM, Sandoval GP, Ballesteros LE. Theories on otic symptoms in temporomandibular disorders: Past and Present. Int J Morphol 2005; 23: 141-56.

2. Ramirez LM, Sandoval GP, Ballesteros LE. Temporomandibular disorders: referred cranio-cervicofacial clinic. Med Oral Patol Oral Cir Bucal 2005 Apr 1;10 Suppl 1: E18-26.

3. YounIss $\mathrm{S}$. The relationship between craniomandibular disorders and otitis media in children. Cranio 1991; 9: 169-73.

4. Klockhoff I, Lindholm L, Westerberg CE. Spontaneous impedance fluctuation-a "tensor tympani syndrome" with special reference to tension headache. Nord Med 1971; 85: 577.

5. Lam DK, Lawrence HP, Tenenbaum HC. Aural symptoms in temporomandibular disorder patients attending a orofacial pain unit. J Orofac Pain 2001; 15: 146-57.

6. CoOper BC, CoOper DL. Recognizing otolaryngologic symptoms in patients with temporomandibular disorders. Cranio 1993; 11: 260-7.

7. PARKer WS, ChOle RA. Tinnitus, vertigo and temporomandibular disorders. Am J Orthod Dentofacial Orthop 1995; 107: 153-8
Es imperativo comprender la necesidad de un manejo interdisciplinario entre el médico y el odontólogo especialista en dolor craneofacial. Igualmente procurar por una visión conservadora en el tratamiento de las tensiones musculares de estos músculos masticatorios y auditivos. Los especialistas en una sola disciplina, no siempre pueden de manera individual resolver la sintomatología presente en un paciente, sin el inestimable sustento de un manejo multidisciplinario. Cada especialidad contribuye en su conocimiento específico al proceso de diagnóstico diferencial que orienta un correcto plan de tratamiento. El éxito clínico depende, por lo tanto, de la habilidad de cada especialista para analizar los diferentes aspectos del mismo problema. La estructura del trabajo en equipo puede ser la mejor opción para mejorar el estado funcional del sistema estomatognático.

8. Rubinstein B. Tinnitus and craniomandibular disorders - Is there a link? Swed Dent J Suppl 1993; 95: 1-46.

9. Chole RA, Parker WS. Tinnitus and vertigo in patients with temporomandibular disorders. Arch Otolaryngol Head Neck Surg 1992; 118: 817-21.

10. Bush FM. Tinnitus and Otalgia in temporomandibular disorders. J Prosthet Dent 1987; 58: 495-8.

11. Pergamalian A, Rudy Te, Zaki HS, Greco CM. The association between wear facets, bruxism, and severity of facial pain in patients with temporomandibular disorders. J Prosthet Dent 2003; 90: 194-200.

12. Bouchet A, Cullleret J. Anatomía descriptiva. Buenos Aires: Editorial Médica Panamericana Vol 8. 1979 , p. 327.

13. Mrrhaug $H$. The incidence of the ear symptoms in cases of malocclusion and temporomandibular joint disturbances. Br J Oral Maxillofac Surg 1964; 2: 28-32.

14. Bernstein JM, Mohl ND, Spiller H. Temporomandibular joint dysfunction masquerading as disease of ear, nose and throat. Trans Am Acad Ophthalmol Otolaryngol 1969; 73: 1208-17.

15. Manni A, Brunori P, Giuliani M, Modoni M, Bizzi G. Oto-vestibular symptoms in patients with tempo- 
romandibular joint dysfunction. Electromyographic study. Minerva Stomatol 1996; 45: 1-7.

16. Ciancaglini R, Loreti P, Radaelli G. Ear, nose and throat symptoms in patients with TMD: The association of symptoms according to severity of arthropathy. J Orofac Pain 1994; 8: 293-7.

17. ECKERDAL O. The petrotynpanic Fissure: A link connecting the tympanic cavity and the temporomandibular joint. Cranio 1991; 9: 15-21.

18. Schames J, Schames M, King E, Ulansey S, Boyd J, Schames E. Trigeminal Pharyngioplasty: Treatment of the forgotten accessory muscles of mastication which are associated with orofacial pain and ear symptomatology. AJPM 2002; 12: 102-12.

19. KLockhoff I. Middle ear muscle reflexes in man. Acta Otolaryngol Suppl (Stockb) 1961; 164: 1-92.

20. Arlen H. The otomandibular syndrome: A new concept. Ear Nose Throat J 1977; 56: 60-2.

21. Ogutcen-Toller M, Juniper RP. Audiological evaluation of the aural symptoms in temporomandibular joint dysfunction. J Craniomaxillofac Surg 1993; 21: 2-8.

22. Travell JG, Simons DG. Dolor y disfunción miofascial. El manual de los puntos gatillo, Mitad superior del cuerpo. Madrid: Editorial Panamericana Vol 1, 2 $2^{\text {a }}$ ed. 2002, pp. 419, 476

23. Virtanen H. Objective tubal tinnitus: a report of two cases. J Laryngol Otol 1983; 97: 857-62.

24. Cohen D, Perez R. Bilateral myoclonus of the tensor tympani: a case report. Otolaryngol Head Neck Surg. 2003; 128: 441.

25. Oliveira CA, Negreiros J, Cavalcante IC, Bahmad F, Venosa AR. Palatal and middle-ear myoclonus; a cause for objective tinnitus. Int Tinnitus J2003; 9: 37-41.

26. Bento RF, Sanchez TG, Miniti A, Tedesco-Marchesi A. Continuous, high-frequency objective tinnitus caused by middle ear myoclonus. Ear Nose Throat J 1998; 77: 814-8.

27. Zipfel TE, Kaza SR, Greene JS. Middle-ear myoclonus. J Laryngol Otol 2000; 114: 207-9.

28. AbDul-Baqi KJ. Objective high-frequency tinnitus of middle-ear myoclonus. J Laryngol Otol 2004; 118: 231-3

29. Remley KB, Coit We, Harnsberger HR, Smoker WR, Jacobs JM, McIff EB. Pulsatile tinnitus and the vascular tympanic membrane: CT, MR, and angiographic findings. Radiology 1990; 174: 383-9.

30. Sila CA, Furlan AJ, Little JR. Pulsatile tinnitus. Stroke 1987; 18: 252-6.
31. Van den Berge H, Kingma H, Kluge C, Marres EH. Electrophysiological aspects of the middle ear muscle reflex in the rat: latency, rise time and effect on sound transmission. Hear Res 1990; 48 : 209-19.

32. Jung HH, Han SH, Nam SY, Kim YH, Kim JL. Myosin heavy chain composition of rat middle ear muscles. Acta Otolaryngol 2004; 124: 569-73.

33. Ochi K, Ohashi T, Kinoshita H. Acoustic tensor tympani response and vestibular-evoked myogenic potential. Laryngoscope 2002; 112: 2225-9.

34. Stach BA, Jerger JF, Jenkins HA. The human acoustic tensor tympani reflex. A case report. Scand Audiol 1984; 13: 93-9.

35. Ried E, Ojeda JP, Agurto M, Ried E, Martinez C. Inverted acoustic reflex in patients with otosclerosis. Acta Otorrinolaringol Esp 2000; 51: 463-7.

36. Shapiro HH, Truex RC. The temporomandibular joint and the auditory function. J Am Dent Assoc 1943; 30: 1147-68.

37. Pau HW, Punke C, Zehlicke T, Dressler D, Sievert U. Tonic contractions of the tensor tympani muscle: a key to some non-specific middle ear symptoms? Hypothesis and data from temporal bone experiments. Acta Otolaryngol 2005; 125 : 1168-75.

38. Franz P, Hamzavi JS, Schneider B, Ehrenberger K. Do middle ear muscles trigger attacks of Ménière's disease? Acta Otolaryngol 2003; 123: 133-7.

39. Klockhoff I, Anderson H. Reflex activity in the tensor tympani muscle recorded in man; preliminary report. Acta Otolaryngol 1960; 51: 184-8.

40. KAMERER DB. Electromyographic correlation of tensor tympani and tensor veli palatini muscles in man. Laryngoscope 1978; 88: 651-62.

41. GraY LP. The relationship between the "superior constrictor swallow" clicking of the ears and ear disease. J Laryngol Otol 1983; 97: 1121-8.

42. Rood SR, Doyle WJ. Morphology of the tensor veli palatiny, tensor tympani and dilatator tubae muscles. Ann Otol Rhinol Laryngol 1978; 87: 202-10.

43. Rood SR. The morphology of muscle tensor veli palatini in the five month human fetus. Am J Anat 1973; 138: 191-5.

44. Su CY, Hsu SP, Chee EC. Electromyographic recording of tensor and levator veli palatini muscle: a modified transnasal insertion method. $\mathbf{L a}$ ryngoscope 1993; 103: 459-62. 
45. SALEN B, Zakrisson JE. Electromyogram of the tensor tympani muscle in man during swallowing. Acta Otolaryngol 1978; 85: 453-5.

46. Barsoumian R, Kuehn DP, Moon JB, Canady JW. An anatomic study of the tensor veli palatini and dilatator tubae muscles in relation to eustachian tube and velar function. Cleft Palate Craniofac J 1998; 35: 101-10.
47. Kierner AC, Mayer R, v Kirschhofer K. Do the tensor tympani and tensor veli palatini muscles of man form a functional unit? A histochemical investigation of their putative connections. Hear Res 2002; 165: 48-52.

48. Malkin DP. The role of TMD dysfunction in the etiology of middle ear diseases. Int J Orthod 1987; 25: 21-1. 